\title{
Alignment control of GEO 600
}

\author{
H Grote ${ }^{1}$, G Heinzel ${ }^{1}$, A Freise ${ }^{1}$, S Goßler ${ }^{1}$, B Willke ${ }^{1}$, H Lück ${ }^{1}$, H Ward ${ }^{2}$, \\ M M Casey ${ }^{2}$, K A Strain ${ }^{2}$, D I Robertson ${ }^{2}$, J Hough ${ }^{2}$ and K Danzmann ${ }^{1}$ \\ ${ }^{1}$ Max-Planck-Institute for Gravitational Physics (Albert-Einstein-Institute) and University of \\ Hannover, Callinstr. 38, D-30167 Hannover, Germany \\ 2 Department of Physics and Astronomy, University of Glasgow, Glasgow G12 8QQ, UK \\ E-mail: hrg@mpq.mpg.de
}

Received 28 August 2003

Published 3 February 2004

Online at stacks.iop.org/CQG/21/S441 (DOI: 10.1088/0264-9381/21/5/009)

\begin{abstract}
We give an overview of the automatic mirror alignment system of the gravitational wave detector GEO 600. In order to achieve the required sensitivity of the Michelson interferometer, the axes of interfering beams have to be superimposed with a residual angle of the order $10^{-8} \mathrm{rad}$. The beam spots have to be centred on the mirrors to minimize coupling of alignment noise into longitudinal signals. We present the actual control topology and results from the system in operation, which controls all alignment degrees of the power-recycled Michelson. With this system continuous lock stretches of more than $121 \mathrm{~h}$ duration were achieved.
\end{abstract}

PACS numbers: $04.80 . \mathrm{Nn}$, 07.60.Ly, 95.55.Ym

(Some figures in this article are in colour only in the electronic version)

\section{Introduction}

Any interferometer can work correctly only if the interfering beams are properly aligned. Even in small-scale (e.g., table-top) experiments, acquisition of initial alignment and compensation of various drifts are challenging tasks for the experimentator. For large-scale interferometers such as GEO 600 [1] with its mirrors suspended as multiple pendulums which need to maintain their alignment for weeks, an automatic alignment system is indispensable. The cavity- and beam-steering mirrors within the vacuum system are suspended as pendulums for the purpose of seismic isolation. While this gives good attenuation of longitudinal and angular motions of the suspended components in the measurement band for gravitational waves $(50 \mathrm{~Hz}-5 \mathrm{kHz})$, the motions are enhanced at the pendulum resonance frequencies (typically around $1 \mathrm{~Hz}$ ) and the components are subject to drifts. The most important task of an automatic angular alignment system is to superimpose the propagation axes of interfering beams. The axes of optical cavities have to match the axes of their input beams and the beam axes of the two long Michelson arms have to match each other at the Michelson output port. In particular, the 
superposition of beam axes minimizes coupling of beam alignment and geometry fluctuations into light power. The second goal of an automatic alignment system is to centre all beam spots on mirrors in order to minimize coupling of mirror alignment noise into longitudinal signals.

Control signals for these alignment tasks are obtained by two methods within GEO 600: for superimposing two beam axes we use the differential wavefront sensing (DWS, see, e.g., [2-4]) technique, which senses the angles between interfering wavefronts. Beam axes have to be superimposed in the cases of the two GEO 600 modecleaners, the power-recycling cavity, the Michelson interferometer and the signal-recycling cavity. To control beam spot positions on mirrors, the small fraction of light that is transmitted through the mirror is detected by a spot position sensing device, for example a quadrant photodiode.

In this paper we briefly describe the standard DWS sensing setup used in GEO 600 with the example of a GEO modecleaner. The design and operation of a DWS feedback loop is shown in some detail for the case of the Michelson interferometer, and a brief overview of the computer control system is given. Throughout this paper the terms 'yaw' and 'pitch' are used to describe angular degrees of freedom of a mirror. A mirror yaw motion results in a horizontal misalignment of a reflected beam, while a pitch motion changes the vertical direction of a reflected beam.

\section{Automatic alignment of a GEO 600 modecleaner}

GEO 600 uses two sequential triangular modecleaners [5] with each cavity mirror being suspended individually by a double pendulum structure. Figure 1 shows the experimental setup for the automatic alignment system of the first modecleaner of GEO 600. The laser beam incident on MMC1a is phase modulated with a radio frequency of $25.2 \mathrm{MHz}$ by the electro-optic modulator EOM1, to allow the use of the Pound-Drever-Hall [6] locking and differential wavefront sensing. The free spectral range of $\mathrm{MC} 1$ is $37.48 \mathrm{MHz}(37.16 \mathrm{MHz}$ for $\mathrm{MC2}$ ) such that the sidebands at $25.25 \mathrm{MHz}$ from the carrier are off resonance.

Beam $E_{\mathrm{i}}+E_{\mathrm{c}}$ leaving mirror MMC1a (downwards in figure 1) consists of the interfering fields of the directly reflected part of the input beam $\left(E_{\mathrm{i}}\right)$ and the field leaking out of the cavity $\left(E_{\mathrm{c}}\right)$.

For the Pound-Drever-Hall sensing, the phase difference between the wavefronts of $E_{\mathrm{i}}$ and $E_{\mathrm{c}}$ is read out over the whole cross section of the interference pattern. The resulting signal is a measure for the match between the incident laser frequency and one of the cavity resonant frequencies. Usually an appropriate feedback loop is used to keep the difference of these frequencies close to zero, what is referred to as 'locking' of the cavity. If the cavity is locked (which is assumed for all following discussions), the DWS method can measure the angle between the wavefronts of $E_{\mathrm{i}}$ and $E_{\mathrm{c}}$ by using a split photodiode and calculating the difference between the two demodulated photocurrents of the different photodiode sections. With a quadrant photodiode this can be done for the horizontal and vertical directions simultaneously. Due to the properties of Gaussian beams, the angle between the wavefronts is generally not identical with the angle between the axes of beams $E_{\mathrm{i}}$ and $E_{\mathrm{c}}$. But if the wavefront angles are measured at two different distances from the beam waist (which is located between mirrors MMC1a and MMC1c, see figure 1), four different quantities can be obtained, giving full information about angular and parallel displacements of the cavity axis against the axis of the incoming beam.

Following this scheme, beam $E_{\mathrm{i}}+E_{\mathrm{c}}$ in figure 1 is split into two paths by the power beamsplitter BS1 which has a transmission of about $50 \%$. The components G1 and G2 are galvanometer scanners with the purpose of centring the beams on the quadrant photodetectors D1 and D2, respectively. The corresponding control loops have a bandwidth of about $1 \mathrm{kHz}$ 


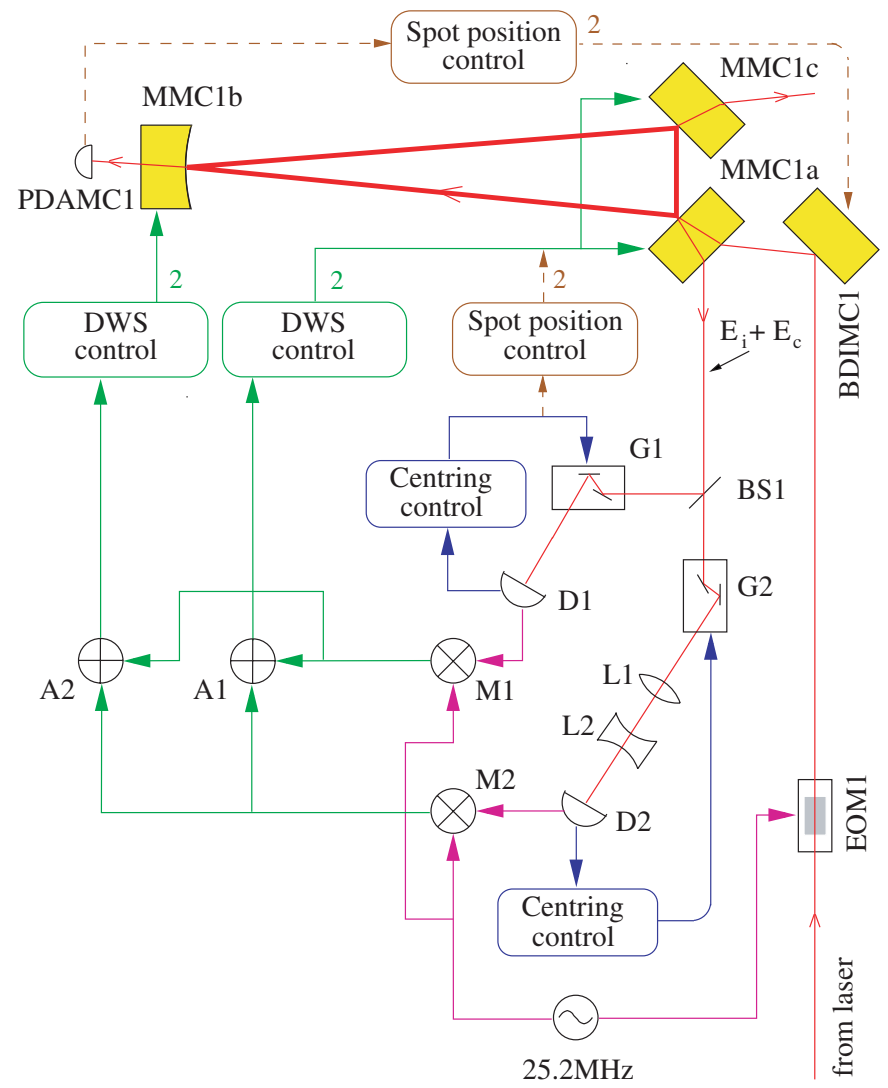

Figure 1. Schematic overview of the alignment control for one modecleaner. Details of the purpose of lenses L1 and L2, galvanometer scanners G1 and G2, photodiodes D1 and D2, mixers M1 and M2 and adders A1 and A2 are given in the text. The numbers next to the control boxes denote the number of degrees of freedom controlled by each path.

and are denoted the centring control. The centring control loops ensure correct DWS signals over a large dynamic range of the initial and residual alignment deviation.

Lenses L1 and L2 in the light path to D2 are used to project beam $E_{\mathrm{i}}+E_{\mathrm{c}}$ into the far field (i.e., adjust an additional Guoy phase shift between the $\mathrm{TEM}_{00}$ and $\mathrm{TEM}_{01 / 10}$ modes close to $90^{\circ}$ ). Each of the detectors D1 and D2 consists of a quadrant photodiode with associated electronics to yield information about the beam spot position, while the angle between the interfering wavefronts (the DWS signal) is available after coherent demodulation of the RF component from each quadrant's photocurrent performed by mixers M1 and M2. Appropriate linear combinations of the DWS signals are then processed by analogue electronic circuits A1 and A2, to generate feedback signals to individual mirrors. Amplified with an appropriate gain and frequency response, these signals are fed back to mirrors MMC1a, MMC1b and MMC1c. Currently we use feedback by analogue electronics with a bandwidth around $0.2 \mathrm{~Hz}$.

The dashed lines show slow digital feedback loops being used for the spot position control. The spot position on mirror MMC1b is controlled by feeding back the PDAMC1 signals to steering mirror BDIMC1, keeping the beam centred on mirror MMC1b (this is possible, as the DWS control has a higher bandwidth than the spot position control, such that the cavity axis follows the axis of the incident beam). The direction of the beam $E_{\mathrm{i}}+E_{\mathrm{c}}$ yields a control 


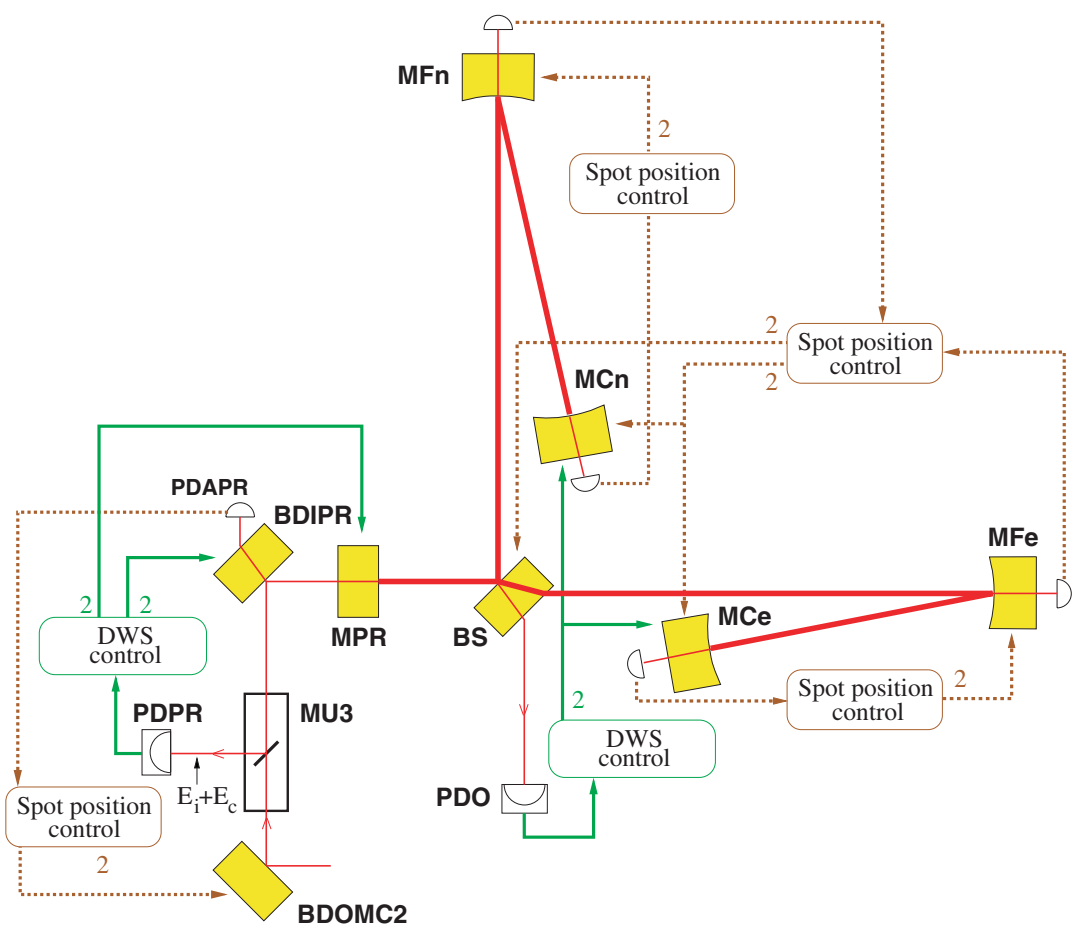

Figure 2. Schematic overview of the alignment control for the power-recycling cavity and Michelson interferometer. PDPR detects the light reflected from MPR and represents a complete DWS alignment sensing setup for four degrees of freedom, while detector PDO at the Michelson output port represents a DWS sensing system for two degrees of freedom. The spot position control topology shown is a simplified version.

signal for the two remaining degrees of freedom of the three cavity mirrors. As this beam is centred on D1 by G1 with high bandwidth $(1 \mathrm{kHz})$, the information about the direction of beam $E_{\mathrm{i}}+E_{\mathrm{c}}$ is contained in the feedback to G1. This information is fed back to MMC1a and MMC1c by another spot position control loop.

The alignment setup for the second GEO 600 modecleaner is identical (except for a phase modulation frequency of $13 \mathrm{MHz}$ instead of $25.25 \mathrm{MHz}$, which is applied by another EOM in the beam path between MC1 and MC2). The complete modecleaner alignment system is supervised by computer programs based on LabView (see section 3.1 for the similar Michelson DWS control), which enables long-term stable operation of the modecleaners without human intervention being required. More details about the modecleaner alignment system (e.g., about the quadrant sensor design and the servo design and performance) can be found in [7].

\section{Automatic alignment of the power-recycled Michelson}

Figure 2 gives a brief overview of the alignment system for the power-recycled Michelson interferometer (without signal recycling).

The Michelson interferometer consisting of beamsplitter BS, folding mirrors MFe, MFn, and end mirrors MCe and MCn forms the power-recycling cavity together with the power-recycling mirror (MPR). In a simple scenario the power-recycling cavity can be seen as a two-mirror cavity, consisting of MPR and the Michelson operating at the dark fringe. This 
cavity has four alignment degrees of freedom determining the beam axis of the power-recycling cavity against the input beam. Although we have an east and north arm of the Michelson, the axis of the power-recycling cavity between MPR and BS is given by the 'average' position of the two beam axes in the long arms.

The most important alignment task for the power-recycling cavity is to superimpose the power-recycling cavity axis (simply called the cavity axis here) on the axis of the beam incident on MPR from the west (called the input axis). The input axis is defined by the alignment of mirrors BDOMC2 and BDIPR. Superimposing these axes is done with the differential wavefront sensing method, sensing the beam reflected from MPR.

The light incident on the power-recycling cavity via BDOMC2 and BDIPR is partly reflected by MPR and interferes with the beam leaking out of the power-recycling cavity. The combined beam $E_{\mathrm{i}}+E_{\mathrm{c}}$ is extracted by a Faraday isolator on the suspended mounting unit MU3 [8] and detected by PDPR. Detector PDPR represents a complete DWS alignment setup for four degrees of freedom similar to the DWS setup for one modecleaner.

The DWS feedback for the power-recycling cavity is applied by a set of coil/magnet actuators mounted at the mirror level of the steering mirror BDIPR and the power-recycling mirror. The performance of this feedback system with a bandwidth of $10 \mathrm{~Hz}$ has been shown in [9].

If the DWS feedback system for the power-recycling cavity is working, there are two degrees of freedom left to be controlled by differential wavefront sensing. The beam axes of the east and north arm have to be superimposed at the main output of the Michelson, south of the beamsplitter, which is referred to as Michelson alignment. Michelson alignment deviations and fluctuations result in a contrast degradation of the dark fringe, which leads to power loss, a smaller Michelson signal (containing the differential length information), and larger couplings of various noise sources to the Michelson signal.

The actuators used for controlling the Michelson alignment are $\mathrm{MCe}$ and $\mathrm{MCn}$ in a differential alignment mode, which does not affect the alignment of the power-recycling cavity. All mirrors that are a part of the Michelson are suspended as triple pendulums with the possibility of applying slow alignment control at the highest mass in the suspension chain. In addition to this, MCe and MCn are equipped with another triple pendulum suspension chain, mounted in parallel with the mirror chain, providing a seismically isolated reaction mass system with coil/magnet actuators at the mass above the mirror ('intermediate mass'), and electrostatic drives at the mirror level. Because of the limited alignment range of the electrostatic drives (to date about $2 \times 10^{-9} \mathrm{rad}$ at $10 \mathrm{~Hz}$ ), we currently use only the intermediate mass (IM) feedback for the Michelson alignment control. Alignment feedback by the electrostatic drives will be added to this, when the Michelson DWS signal has reached its final low noise level.

The loop filters for the IM feedback have to provide about $200^{\circ}$ of phase lead around the unity gain frequency of $10 \mathrm{~Hz}$, to yield a stable feedback system. As the gain of these filters above $10 \mathrm{~Hz}$ can be very high, care has to be taken concerning saturation of these filters. Figure 3 shows the simulated open loop gain and phase of the initial implementation of the Michelson fast autoalignment for pitch (the filter- and actuator-transfer function combined). A unity gain frequency of about $10 \mathrm{~Hz}$ can be achieved with this design. The loop is only stable and robust for unity-gain frequencies between 5 and $10 \mathrm{~Hz}$. As the resonance frequencies do not differ much between MCe and MCn, only one filter was used, to control pitch (and another filter to control yaw) of both mirrors.

After the Michelson is locked longitudinally, the Michelson DWS fast feedback can be switched on. However, without additional care, the acquisition of the DWS fast feedback is critical. If the deviation from perfect Michelson alignment at the moment of closing the alignment loop is too large, locking of the alignment loop is not achieved as the 


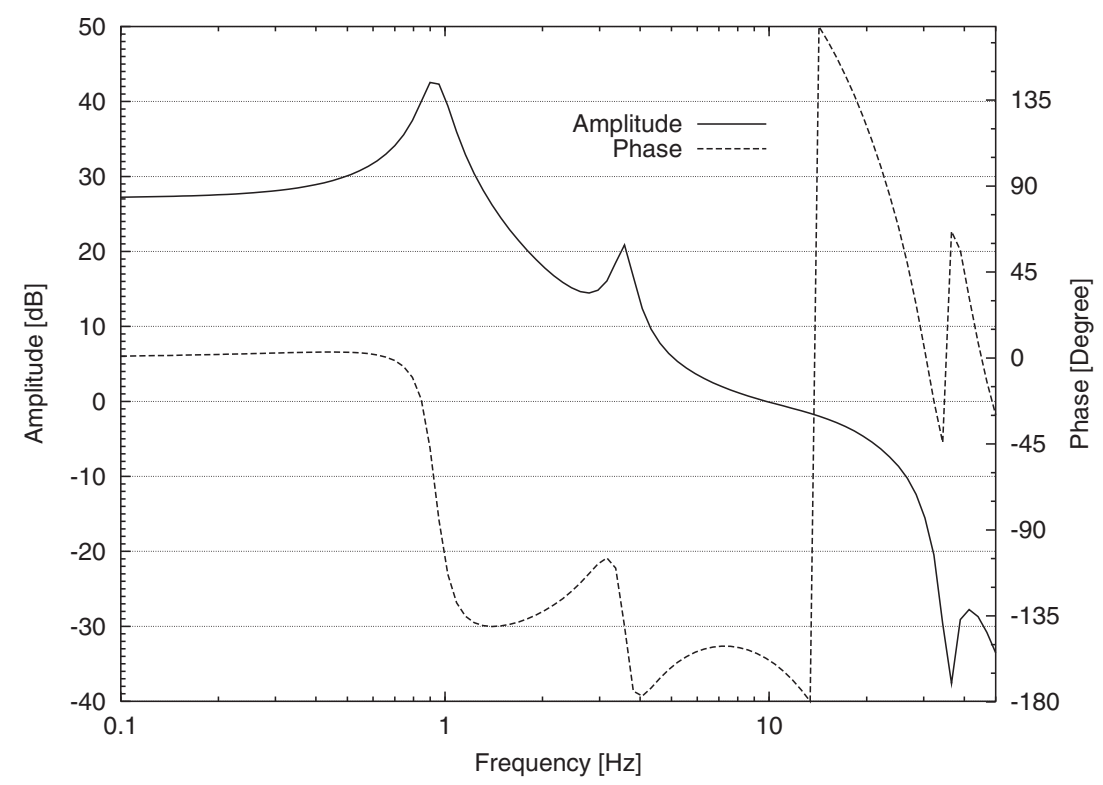

Figure 3. Simulated open loop gain and phase of the Michelson fast autoalignment for pitch.
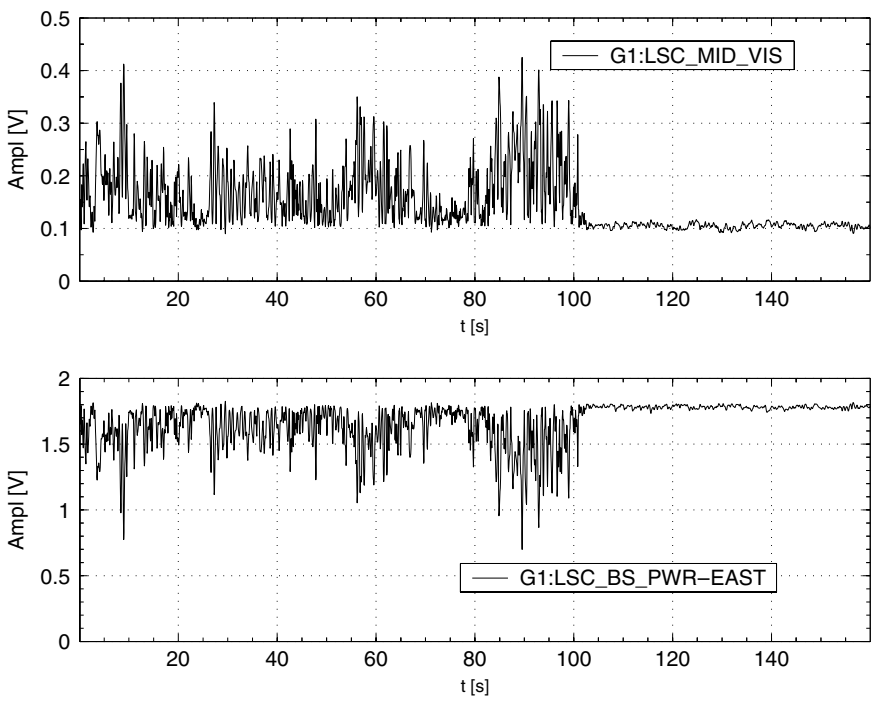

Figure 4. Time series of Michelson interferometer power levels with the Michelson DWS control switched on around $t=100 \mathrm{~s}$. The upper trace shows the light power at the Michelson output port, the lower trace displays the power in the east arm of the Michelson. Without autoalignment, the interferometer is almost unstable in this configuration.

Michelson alignment gets disturbed too much during initial alignment oscillations, such that the longitudinal lock is lost.

For acquiring lock safely, it is advantageous to switch the Michelson DWS feedback on at the same time as the longitudinal feedback at the intermediate mass stage is switched on. Additionally, the gain of the Michelson DWS loop is ramped by hardware from zero to its 


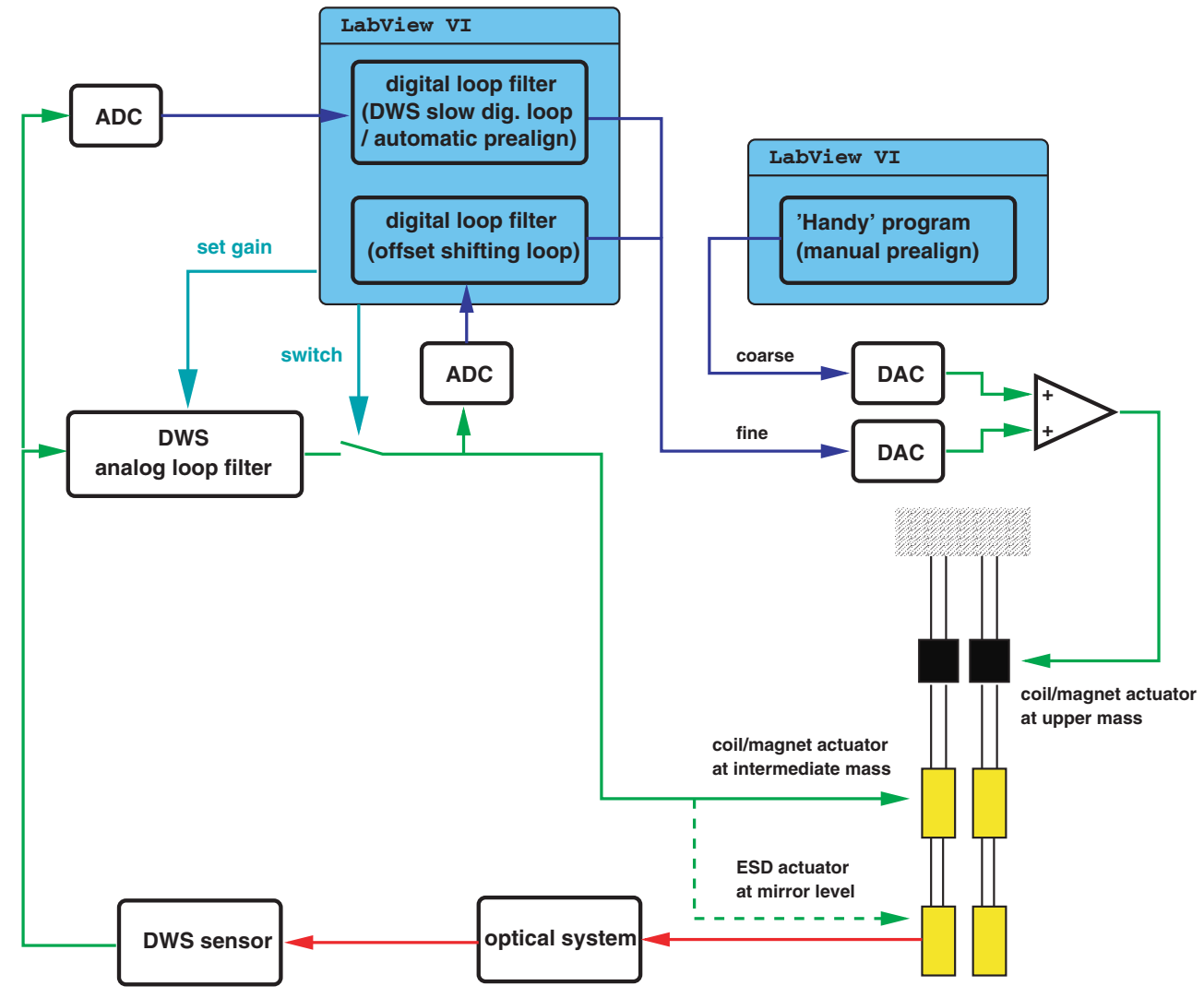

Figure 5. Integration of the LabView-based computer control with the DWS system for the Michelson alignment. Depending on the detector state, the DWS feedback is switched on and off automatically.

nominal value over approximately $1 \mathrm{~s}$, to enable a soft acquisition of the system (for this short period the DWS loop remains stable even with low gain). For a stable operation of the Michelson DWS feedback, with a bandwidth around $10 \mathrm{~Hz}$, it was necessary to implement notch filters for the vertical suspension modes around 22 and $32 \mathrm{~Hz}$, which otherwise lead to instability. Further critical parameters for the stability of the Michelson DWS feedback are the beam spot positions on the four end mirrors. In particular, the spot positions on mirrors MCe and MCn are important, as these mirrors are used for the DWS feedback. If a spot is not centred on a mirror here, the coupling of angular mirror motions (e.g., caused by the DWS feedback) to longitudinal displacement is altered. If the bandwidth of the Michelson longitudinal feedback loop is low, there can be a coupled oscillation of the DWS and longitudinal feedback loop. Only after these spot positions were fixed by spot position control loops could continuous locking times exceed many hours.

Figure 4 shows a time series of the light power at the dark port (upper trace) and the power in the east arm of the Michelson (lower trace). At $t=100 \mathrm{~s}$ the DWS alignment feedback of the Michelson is switched on. The remaining Michelson alignment noise with DWS feedback active corresponds to roughly $5 \times 10^{-8} \mathrm{rad}$. This needs to be improved by a factor of 5 , in order to meet the required alignment noise limit of $1 \times 10^{-8} \mathrm{rad}$. However, it is not expected to be difficult to reach this goal once the electrostatic drives are used for DWS feedback in conjunction with the coil/magnet DWS feedback at the intermediate masses. 

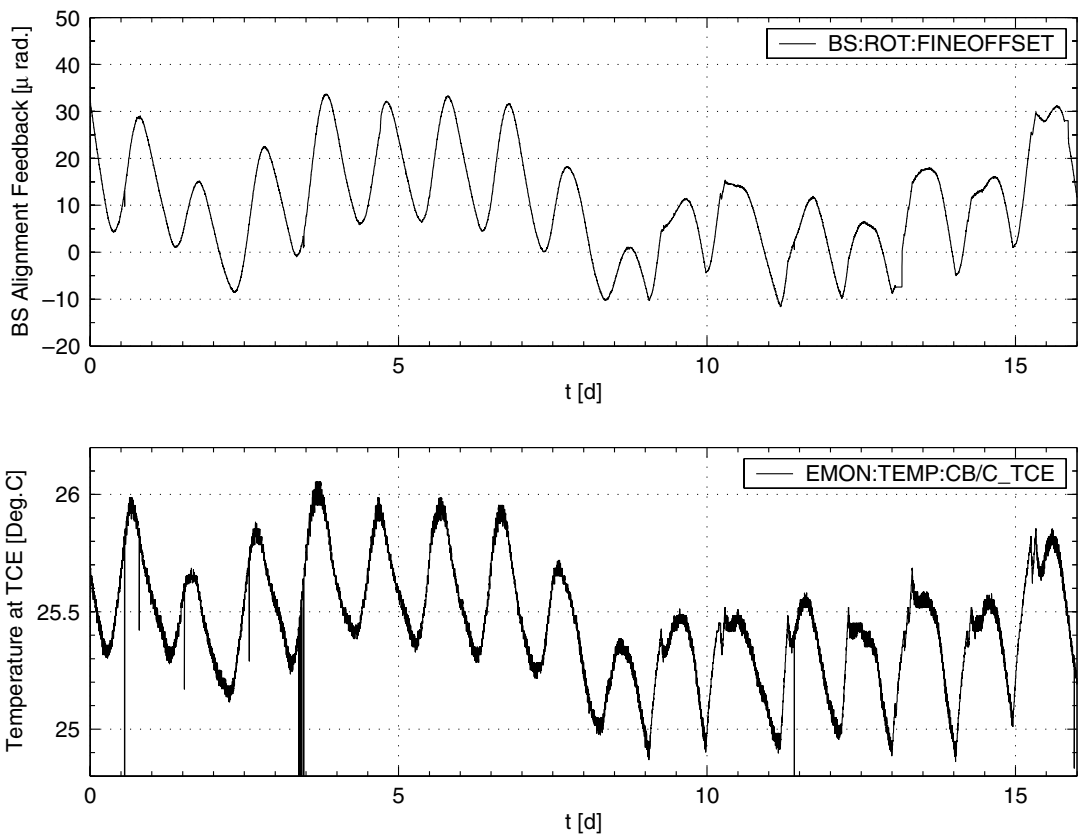

Figure 6. Beamsplitter yaw alignment feedback (top) and central cluster temperature (bottom) over 16 days of the ' $\mathrm{S} 1$ ' run.

\subsection{Computer control}

A computer control system based on LabView is used to monitor signals of the analogue electronics by means of programs called 'virtual instruments' (VI) [10]. The gains of the servo loops can be set with this system and the feedback loops can be switched on and off automatically, depending on the state of the detector. The spot position controls (as shown by the dashed lines in figure 2) are implemented as digital feedback loops with a bandwidth around $0.1 \mathrm{~Hz}$ by virtual instruments as well.

Figure 5 gives an overview of the VIs controlling the Michelson DWS feedback. Manual coarse alignment of each mirror is done with a dedicated VI, applying offsets to the upper mass of the suspension chain. These offsets are not altered during normal operation. For test purposes, the Michelson DWS feedback can be used in a digital only mode with low bandwidth. In the standard mode, however, the DWS analogue feedback applied to the intermediate mass is monitored and held close to zero, by adding digitally controlled offsets with high resolution to the upper mass stage ('offset shifting loop'). This split feedback system enhances the dynamic range and ensures a close to perfect aligned interferometer even after a loss of lock, when the analogue DWS feedback is switched off.

\subsection{Long-term operation}

During the data taking run in August/September 2002 ('S1' run), the interferometer was continuously operated for 16 days with a duty cycle of more than $98 \%$. With the exception of a severe misalignment caused by an earthquake in Italy, the Michelson could relock automatically after losses of lock. As an example of the free mirrors alignment motion, figure 6 shows the beamsplitter yaw alignment feedback (top) and the central cluster temperature (bottom) during this period. The alignment fluctuations of the virtually 
uncontrolled mirror (measured by the applied feedback) are about $50 \mu \operatorname{rad}_{\mathrm{pp}}$. Without alignment control this would lead to a spot position deviation of $60 \mathrm{~mm}_{\mathrm{pp}}$ on the far end mirrors MCe and MCn. It can be seen that the alignment signal is strongly dominated by the temperature in the central cluster, which is dominated by a period of $24 \mathrm{~h}$.

\section{Conclusion}

The complete automatic alignment system for the two suspended ring-type modecleaners and the power-recycled Michelson interferometer of GEO 600 is installed and successfully operating. The modecleaners have been in nearly continuous operation since spring 2001 and have required almost no manual mirror alignment.

GEO's power-recycled Michelson interferometer is equipped with a complete automatic alignment system controlling the two alignment degrees of freedom for each suspended mirror. This alignment system has been found to be indispensable for stable detector operation. During the S1 data taking run in August 2002, a duty cyle of more than 98\% was obtained with the longest continuous stretch of lock of more than $121 \mathrm{~h}$ duration. Further, the alignment system reduces the coupling of various noise sources (such as, e.g., laser power noise) into the gravitational wave signal.

\section{Acknowledgments}

The authors would like to thank PPARC in the UK, the BMBF and the state of Lower Saxony in Germany. We also thank the whole GEO 600 team for support of this work.

\section{References}

[1] Willke B et al 2002 The GEO 600 gravitational wave detector Class. Quantum Grav. B 19 1377-87

[2] Morrison E, Meers B J, Robertson D I and Ward H 1994 Automatic alignment of optical interferometers Appl. Opt. 33 5041-9

[3] Heinzel G, Rüdiger A, Schilling R, Strain K A, Winkler W, Mizuno J and Danzmann K 1999 Automatic beam alignment in the Garching 30-m prototype of a laser-interferometric gravitational wave detector Opt. Commun. $160321-34$

[4] Heinzel G 1999 Advanced optical techniques for laser-interferometric gravitational-wave detectors PhD Thesis University of Hannover (also available as MPQ report 243) pp 141-6

[5] Goßler S et al 2003 The GEO 600 mode-cleaning and injection optics Rev. Sci. Instrum. 743787

[6] Drever R W P, Hall J L, Kowalski F V, Hough J, Ford G M, Munley A J and Ward H 1983 Laser phase and frequency stabilisation using an optical resonator Appl. Phys. B 31 97-105

[7] Grote $\mathrm{H}$ et al 2003 Automatic beam alignment for the modecleaner cavities of GEO $600 \mathrm{Appl}$. Opt. at press

[8] Freise A et al 2002 Performance of a 1200 m long suspended optical cavity Class. Quantum Grav. B 19 1389-97

[9] Grote H et al 2002 The automatic alignment system of GEO 600 Class. Quantum Grav. B 19 1849-55

[10] Casey M M, Ward H and Robertson D I 2000 Computer monitoring and control of the GEO 600 gravitational wave detector Rev. Sci. Instrum. 71 3910-7 